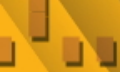

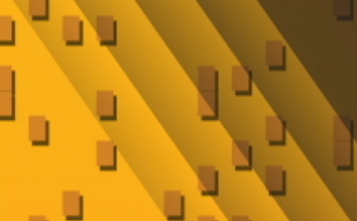

$$
\begin{aligned}
& \text { AVgebra \& } \\
& \text { Number } \\
& \text { Theory } \\
& \text { Jalume } 6 \\
& 2012
\end{aligned}
$$

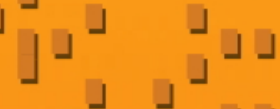

$$
\begin{aligned}
& \text { ل」 }
\end{aligned}
$$

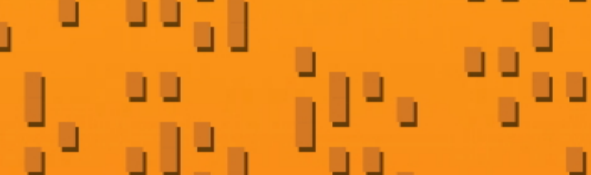

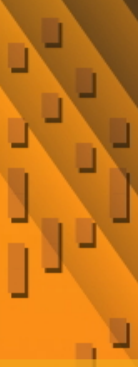

The Chevalley-Shephard-Todd theorem for finite linearly reductive group schemes

Matthew Satriano 


\title{
The Chevalley-Shephard-Todd theorem for finite linearly reductive group schemes
}

\author{
Matthew Satriano
}

\begin{abstract}
We generalize the classical Chevalley-Shephard-Todd theorem to the case of finite linearly reductive group schemes. As an application, we prove that every scheme $X$ which is étale-locally the quotient of a smooth scheme by a finite linearly reductive group scheme is the coarse space of a smooth tame Artin stack (as defined by Abramovich, Olsson, and Vistoli), whose stacky structure is supported on the singular locus of $X$.
\end{abstract}

\section{Introduction}

Given a field $k$ and an action of a finite (abstract) group $G$ on a $k$-vector space $V$, we obtain a linear action of $G$ on the polynomial ring $k[V]$. A central theme in invariant theory is determining when certain nice properties of a ring with $G$-action are inherited by its invariants. In particular, it is natural to ask when $k[V]^{G}$ is polynomial. If $G$ acts faithfully on $V$, we say $g \in G$ is a pseudoreflection (with respect to the action of $G$ on $V$ ) if $V^{g}$ is a hyperplane. The classical ChevalleyShephard-Todd theorem states:

Theorem 1.1 [Bourbaki 1968, §5, Theorem 4]. If $G \rightarrow \operatorname{Aut}_{k}(V)$ is a faithful representation of a finite group and the order of $G$ is not divisible by the characteristic of $k$, then $k[V]^{G}$ is polynomial if and only if $G$ is generated by pseudoreflections.

In this paper we generalize this theorem to the case of finite linearly reductive group schemes. To do so, we first need a notion of pseudoreflection in this setting.

Definition 1.2. Let $k$ be a field and $V$ a finite-dimensional $k$-vector space with a faithful action of a finite linearly reductive group scheme $G$ over $\operatorname{Spec} k$. We say that a subgroup scheme $N$ of $G$ is a pseudoreflection if $V^{N}$ has codimension 1 in $V$. We define the subgroup scheme generated by pseudoreflections to be the intersection of the subgroup schemes which contain all of the pseudoreflections of $G$. We say $G$ is generated by pseudoreflections if $G$ is the subgroup scheme generated by pseudoreflections.

Keywords: Chevalley-Shephard-Todd, pseudoreflection, linearly reductive, tame stacks. 
Over algebraically closed fields, Theorem 1.1 generalizes to

Theorem 1.3. Let $k$ be an algebraically closed field and $V$ a finite-dimensional $k$-vector space with a faithful action of a finite linearly reductive group scheme $G$ over Spec $k$. Then $k[V]^{G}$ is polynomial if and only if $G$ is generated by pseudoreflections.

A more technical version of this theorem holds over fields which are not algebraically closed; however, the "only if" direction does not hold for finite linearly reductive group schemes in general (see Example 2.4). We instead prove the "only if" direction for the smaller class of stable group schemes, which we now define (see Proposition 2.2 for examples). Over an algebraically closed field, the class of stable group schemes coincides with that of finite linearly reductive group schemes. Recall from [Abramovich et al. 2008, Definition 2.9] that $G$ is called well-split if it is isomorphic to a semidirect product $\Delta \rtimes Q$, where $\Delta$ is a finite diagonalizable group scheme and $Q$ is a finite constant tame group scheme; here, tame means that the degree is prime to the characteristic.

Definition 1.4. A group scheme $G$ over a field $k$ is called stable if the following two conditions hold:

(a) for all finite field extensions $K / k$, every subgroup scheme of $G_{K}$ descends to a subgroup scheme of $G$;

(b) there exists a finite Galois extension $K / k$ such that $G_{K}$ is well-split.

Remark 1.5. If $G$ is a finite linearly reductive group scheme over a perfect field $k$, then [Abramovich et al. 2008, Lemma 2.11] shows that condition (b) is automatically satisfied.

Theorem 1.3 is a special case of the following generalization of the ChevalleyShephard-Todd theorem. This is the first main result of this paper.

Theorem 1.6. Let $k$ be a field and $V$ a finite-dimensional $k$-vector space with a faithful action of a finite linearly reductive group scheme $G$ over Spec $k$. If $G$ is generated by pseudoreflections, then $k[V]^{G}$ is polynomial. The converse holds if $G$ is stable.

We also prove a version of this theorem for an action of a finite linearly reductive group scheme on a smooth scheme.

Definition 1.7. Given a smooth affine scheme $U$ over Spec $k$ with a faithful action of a finite linearly reductive group scheme $G$ which fixes a field-valued point $x \in$ $U(K)$, we say a subgroup scheme $N$ of $G$ is a pseudoreflection at $x$ if $N_{K}$ is a pseudoreflection with respect to the induced action of $G_{K}$ on the cotangent space at $x$. We define what it means for $G$ to be generated by pseudoreflections at $x$ in the same manner as in Definition 1.2. 
Theorem 1.6 then has the following corollary.

Corollary 1.8. Let $k$ be a field and let $U$ be a smooth affine $k$-scheme with a faithful action by a finite linearly reductive group scheme $G$ over $\operatorname{Spec} k$. Let $x \in U(K)$, where $K / k$ is a finite separable field extension, and suppose $x$ is fixed by $G$. If $G$ is generated by pseudoreflections at $x$, then $U / G$ is smooth at the image of $x$. The converse holds if $G$ is stable.

The second main result of this paper is this:

Theorem 1.9. Let $k$ be a field and let $U$ be a smooth affine $k$-scheme with a faithful action by a stable group scheme $G$ over Spec $k$. Suppose $K / k$ is a finite separable field extension and $G$ fixes a point $x \in U(K)$. Let $M=U / G$, let $M^{0}$ be the smooth locus of $M$, and let $U^{0}=U \times{ }_{M} M^{0}$. If $G$ has no pseudoreflections at $x$, then after possibly shrinking $M$ to a smaller Zariski neighborhood of the image of $x$, we have that $U^{0}$ is a $G$-torsor over $M^{0}$.

In the classical case, Theorem 1.9 follows directly from Corollary 1.8 and the purity of the branch locus theorem [Grothendieck and Raynaud 1971, X.3.1]. For us, however, a little more work is needed since $G$ is not necessarily étale.

As an application of Theorem 1.9, we generalize the well-known result (see, for example, [Vistoli 1989, (2.9)] or [Fantechi et al. 2007, Remark 4.9]) that schemes with quotient singularities prime to the characteristic are coarse spaces of smooth Deligne-Mumford stacks. We say a scheme has linearly reductive singularities if it is étale-locally the quotient of a smooth scheme by a finite linearly reductive group scheme. We show that every such scheme $M$ is the coarse space of a smooth tame Artin stack (in the sense of [Abramovich et al. 2008]) whose stacky structure is supported at the singular locus of $M$. More precisely,

Theorem 1.10. Let $k$ be a perfect field and $M$ a $k$-scheme with linearly reductive singularities. Then it is the coarse space of a smooth tame stack $\mathfrak{X}$ over $k$ such that $f^{0}$ in the diagram

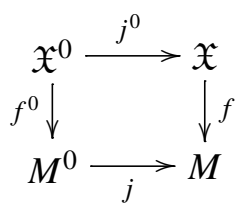

is an isomorphism, where $j$ is the inclusion of the smooth locus of $M$ and $\mathfrak{X}^{0}=$ $M^{0} \times{ }_{M} \mathfrak{X}$.

This paper is organized as follows. In Section 2, we prove the "if" direction of Theorem 1.6 and reduce the proof of the "only if" direction to the special case of Theorem 1.9 in which $U=\mathbb{V}^{\vee}(V)$ for some $k$-vector space $V$ with $G$-action (see the section on notation below). This special case is proved in Section 3. The 
key input for the proof is [Iwanari 2009, Theorem 2.3], which we reinterpret in the language of pseudoreflections. We finish the section by proving Corollary 1.8. In Section 4, we use Corollary 1.8 to complete the proof of Theorem 1.9. In Section 5, we prove Theorem 1.10.

Notation. Throughout this paper, $k$ is a field and $S=\operatorname{Spec} k$. If $V$ is a $k$-vector space with an action of a group scheme $G$, then we denote by $\mathbb{V}^{\vee}(V)$, or simply $\mathbb{V}^{\vee}$ if $V$ is understood, the scheme Spec $k[V]$ whose $G$-action is given by the dual representation on functor points. Said another way, if $G=\operatorname{Spec} A$ is affine and its action on $V$ is given by the coaction map $\sigma: V \longrightarrow V \otimes_{k} A$, then the coaction map $k[V] \longrightarrow k[V] \otimes_{k} A$ defining the $G$-action on $\mathbb{V}^{\vee}$ is given by $\sum a_{i} v_{i} \mapsto \sum a_{i} \sigma\left(v_{i}\right)$.

All Artin stacks $\mathfrak{X}$ in this paper are assumed to have finite diagonal, so that if $\mathfrak{X}$ is locally of finite presentation, it has a coarse space by [Conrad 2004, Theorem 1.1] (see also [Keel and Mori 1997]). Given a locally finitely presented scheme $U$ with an action of a finite flat group scheme $G$, we denote by $U / G$ the coarse space of the stack $[U / G]$.

If $R$ is a ring and $\mathscr{I}$ an ideal of $R$, then we denote by $V(\mathscr{Y})$ the closed subscheme of Spec $R$ defined by $\mathscr{I}$.

\section{Linear actions on polynomial rings}

The "if" direction of Theorem 1.6. Our first goal is to prove the "if" direction of Theorem 1.6. We begin with examples of stable group schemes and with some basic results about the subgroup scheme generated by pseudoreflections.

Lemma 2.1. Suppose $k$ is perfect and $G$ is a finite linearly reductive group scheme over $S$. If the identity component $\Delta$ of $G$ is diagonalizable and $G / \Delta$ is constant, then there exists a finite linearly reductive group scheme $\tilde{G}$ over $\mathbb{Z}$ such that $\tilde{G}_{k}=$ $G$. If $H$ is a closed subgroup scheme of $G$, then there exists a closed subgroup scheme $\tilde{H}$ of $\tilde{G}$ whose pullback to $k$ is $H$. If $H$ is normal in $G$, then $\tilde{H}$ is normal in $\tilde{G}$.

Proof. Let $Q=G / \Delta$. Since $k$ is perfect, the connected-étale sequence

$$
1 \longrightarrow \Delta \longrightarrow G \longrightarrow Q \longrightarrow 1
$$

is functorially split (see [Tate 1997, 3.7 (IV)]). Since $\Delta$ is diagonalizable, it is of the form $\operatorname{Spec} k[A]$, where $A$ is a finitely generated abelian group. Note that as a scheme $G=\Delta \times_{k} Q$ and that its group scheme structure is given by a homomorphism:

$$
\epsilon: Q \longrightarrow \operatorname{Aut}(\Delta)=\operatorname{Aut}(A) .
$$

We can therefore let $\tilde{G}=\operatorname{Spec} \mathbb{Z}[A] \times \mathbb{Z} Q$ with group scheme structure induced by $\epsilon$. 
Now let $H$ be a closed subgroup scheme of $G$. Letting $\Delta^{\prime}=H \cap \Delta$ and $Q^{\prime}=$ $H / \Delta^{\prime}$, we have a commutative diagram

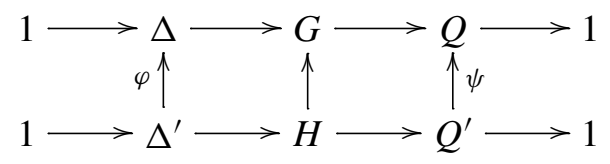

with exact rows. Since $\Delta$ is connected, we see $\Delta^{\prime}$ is the connected component of the identity of $H$. Therefore, the bottom row of the above diagram is the connectedétale sequence of $H$, and so

$$
H=\Delta^{\prime} \rtimes Q^{\prime},
$$

as $k$ is perfect. Since $\Delta^{\prime}$ is diagonalizable and $Q^{\prime}$ is constant, we can define $\tilde{H}$ in the same way we defined $\tilde{G}$.

We now show that $\tilde{H}$ is a closed subgroup scheme of $\tilde{G}$. Let $*$ denote the action of $Q$ (resp. $Q^{\prime}$ ) on $\Delta$ (resp. $\Delta^{\prime}$ ). Since the splitting of the connected-étale sequence of a finite group scheme over a perfect field is functorial, we see that for all $q^{\prime} \in Q^{\prime}$ and local sections $\delta^{\prime}$ of $\Delta^{\prime}$,

$$
\psi\left(q^{\prime}\right) * \varphi\left(\delta^{\prime}\right)=\varphi\left(q^{\prime} * \delta^{\prime}\right) .
$$

We therefore obtain a closed immersion from $\tilde{H}$ to $\tilde{G}$ whose pullback to $k$ is the morphism from $H$ to $G$.

Lastly, we show that if $H$ is normal in $G$, then $\tilde{H}$ is normal in $\tilde{G}$. Let $\Delta^{\prime}=$ Spec $k\left[A^{\prime}\right]$, where $A^{\prime}$ is a finitely generated abelian group. Showing that $\tilde{H}$ is normal in $\tilde{G}$ is equivalent to showing that $Q^{\prime}$ is normal in $Q$, and for all local sections $\delta \in \Delta, \delta^{\prime} \in \Delta^{\prime}, q \in Q$, and $q^{\prime} \in Q^{\prime}$, we have

$$
q *\left(\delta^{-1} \delta^{\prime} \cdot\left(q^{\prime-1} * \delta\right)\right) \in \Delta^{\prime} .
$$

We know that $Q^{\prime}$ is normal in $Q$ as $H$ is normal in $G$. To check the latter statement about local sections, note that it can be reformulated as follows: for every $q \in Q$ and $q^{\prime} \in Q$, the homomorphism

$$
\begin{aligned}
A & \rightarrow A \times A^{\prime} \\
a & \mapsto\left(q *\left(a^{-1} \cdot q^{\prime-1} * a\right), q * \bar{a}\right)
\end{aligned}
$$

factors through $A^{\prime}$; here $\bar{a}$ denotes the image of $a$ under the projection from $A$ to $A^{\prime}$. Since this statement makes no reference to the base scheme, it can be checked over $k$, where the normality of $H$ in $G$ yields the desired factorization.

Proposition 2.2. Let $G$ be a finite group scheme over $S$. Consider the following conditions:

(1) $G$ is diagonalizable. 
(2) $G$ is a constant group scheme.

(3) $k$ is perfect, the identity component $\Delta$ of $G$ is diagonalizable, and $G / \Delta$ is constant.

If any of them holds, then $G$ is stable.

Proof. It is clear that finite diagonalizable group schemes and finite constant group schemes are stable, so we consider the last case. Let $Q=G / \Delta$. Since $k$ is perfect, the connected-étale sequence

$$
1 \longrightarrow \Delta \longrightarrow G \longrightarrow Q \longrightarrow 1
$$

is functorially split. Let $K / k$ be a finite extension and let $H$ be a subgroup scheme of $G_{K}$. Letting $\Delta^{\prime}=H \cap \Delta_{K}$ and $Q^{\prime}=H / \Delta^{\prime}$, we have a commutative diagram

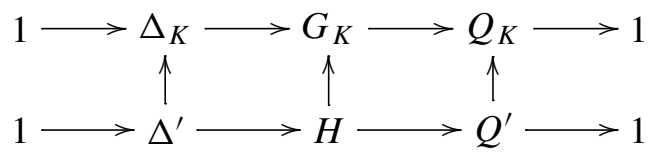

with exact rows. Since $\Delta$ is connected and has a $k$-point, [Grothendieck 1967, 4.5.14] shows that $\Delta$ is geometrically connected. In particular, $\Delta_{K}$ is the connected component of the identity of $G_{K}$, and so $\Delta^{\prime}$ is the connected component of the identity of $H$. Therefore, the bottom row of the above diagram is the connectedétale sequence of $H$. The proposition then follows from Lemma 2.1.

Lemma 2.3. Let $V$ be a finite-dimensional $k$-vector space with a faithful action of a stable group scheme $G$ over $S$, and let $H$ be the subgroup scheme generated by pseudoreflections. If $K / k$ is an algebraic extension of fields, then a subgroup scheme of $G_{K}$ is a pseudoreflection if and only if it descends to a pseudoreflection. Furthermore, $H_{K}$ is the subgroup scheme of $G_{K}$ generated by pseudoreflections.

Proof. Note first that if $P$ is a subgroup scheme of $G_{K}$, then there exists a subgroup scheme $P_{0}$ of $G$ such that $\left(P_{0}\right)_{K}=P$. If $K / k$ is a finite extension, this follows from the fact that $G$ is stable. If $K / k$ is an infinite extension, by a standard limit argument, there exists a finite extension $L / k$ and a subgroup scheme $P_{1}$ of $G_{L}$ such that $\left(P_{1}\right)_{K}=P$. We then obtain our desired $P_{0}$ as $L / k$ is a finite extension. The first claim of the proposition then follows from the fact that

$$
\left(V_{K}\right)^{N_{K}}=\left(V^{N}\right)_{K}
$$

for any subgroup scheme $N$ of $G$. The second claim follows from the fact that if $P^{\prime}$ and $P^{\prime \prime}$ are subgroup schemes of $G$, then $P_{K}^{\prime}$ contains $P_{K}^{\prime \prime}$ if and only if $P^{\prime}$ contains $P^{\prime \prime}$.

We remark that even in characteristic zero, Lemma 2.3 is false for general finite linearly reductive group schemes $G$, as the following example shows. Note that this 
example also shows that the "only if" direction of Theorem 1.6 and of Corollary 1.8 is false for general finite linearly reductive group schemes.

Example 2.4. Let $k$ be a field contained in $\mathbb{R}$ or let $k=\mathbb{F}_{p}$ for $p$ congruent to $3 \bmod 4$. Let $K=k(i)$, where $i^{2}=-1$, and let $G$ be the locally constant group scheme over Spec $k$ whose pullback to Spec $K$ is $\mathbb{Z} / 2 \times \mathbb{Z} / 2$ with the Galois action that switches the two $\mathbb{Z} / 2$ factors. Let $g_{1}$ and $g_{2}$ be the generators of the two $\mathbb{Z} / 2$ factors and consider the action

$$
\rho: G_{K} \longrightarrow \operatorname{Aut}_{K}\left(K^{2}\right)
$$

on the $K$-vector space $K^{2}$ given by

$$
\rho\left(g_{1}\right):(a, b) \mapsto(-b i, a i), \quad \rho\left(g_{2}\right):(a, b) \mapsto(b i,-a i) .
$$

Then $\rho$ is Galois-equivariant and hence comes from an action of $G$ on $k^{2}$. Note that $\mathbb{Z} / 2 \times 1$ and $1 \times \mathbb{Z} / 2$ are both pseudoreflections of $G_{K}$, as the subspaces which they fix are $K \cdot(1, i)$ and $K \cdot(1,-i)$, respectively. Since $G_{K}$ is not a pseudoreflection, it follows that there are no Galois-invariant pseudoreflections of $G_{K}$, and hence, the subgroup scheme generated by pseudoreflections of $G$ is trivial; the subgroup scheme generated by pseudoreflections of $G_{K}$, however, is $G_{K}$.

Corollary 2.5. If $V$ is a finite-dimensional $k$-vector space with a faithful action of a stable group scheme $G$ over $S$, then the subgroup scheme generated by pseudoreflections is normal in $G$.

Proof. We denote by $H$ the subgroup scheme generated by pseudoreflections. Let $T$ be an $S$-scheme and let $g \in G(T)$. We must show the subgroup schemes $H_{T}$ and $g H_{T} g^{-1}$ of $G_{T}$ are equal. To do so, it suffices to check this on stalks and so we can assume $T=\operatorname{Spec} R$, where $R$ is strictly Henselian. By [Abramovich et al. 2008, Lemma 2.17], we need only show that these two group schemes are equal over the closed fiber of $T$, so we can further assume that $R=K$ is a field. Since $G$ is finite over $S$, the residue fields of $G$ are finite extensions of $k$. We can therefore assume that $K / k$ is a finite field extension.

By Lemma 2.3, we know that $H_{K}$ is the subgroup scheme of $G_{K}$ generated by pseudoreflections. Note that if $N^{\prime}$ is a pseudoreflection of $G_{K}$, then $g N^{\prime} g^{-1}$ is as well since

$$
V_{K}^{g N^{\prime} g^{-1}}=g\left(V_{K}^{N^{\prime}}\right)
$$

As a result, $g H_{K} g^{-1}=H_{K}$, which completes the proof.

Lemma 2.6. Given a finite-dimensional k-vector space $V$ with a faithful action of a finite linearly reductive group scheme $G$ over $S$, let $\left\{N_{i}\right\}$ denote the set of 
pseudoreflections of $G$ and let $H$ be the subgroup scheme generated by pseudoreflections. Then

$$
k[V]^{H}=\bigcap_{i} k[V]^{N_{i}} .
$$

Proof. Let $R=\bigcap_{i} k[V]^{N_{i}}$. Consider the functor

$$
\begin{aligned}
F:(k \text {-alg }) & \rightarrow \text { (Groups) } \\
A & \mapsto\left\{g \in G(A) \mid g(m)=m \text { for all } m \in R \otimes_{k} A\right\} .
\end{aligned}
$$

Since each $k[V]^{N_{i}}$ is finitely generated, we see $R$ is as well. Let $r_{1}, \ldots, r_{n}$ be a finite set of generators for $R$. We see then that $F$ is representable by the intersection of the stabilizers $G_{r_{j}}$, and so is a closed subgroup scheme of $G$. Since $F$ contains every pseudoreflection, we see $H \subset F$. We therefore have the containments

$$
R \subset k[V]^{F} \subset k[V]^{H} \subset \bigcap_{i} k[V]^{N_{i}},
$$

from which the lemma follows.

If $N$ is any subgroup scheme of $G$, it is linearly reductive by [Abramovich et al. 2008, Proposition 2.7]. It follows that

$$
V \simeq V^{N} \oplus V / V^{N}
$$

as $N$-representations. If $N$ is a pseudoreflection, then $\operatorname{dim}_{k} V / V^{N}=1$. Let $v$ be a generator of the 1-dimensional subspace $V / V^{N}$ and let $\sigma: V \rightarrow V \otimes_{k} B$ be the coaction map, where $N=\operatorname{Spec} B$. Then via the above isomorphism, $\sigma$ is given by

$$
\begin{aligned}
V^{N} \oplus V / V^{N} & \rightarrow\left(V^{N} \otimes_{k} B\right) \oplus\left(V / V^{N} \otimes_{k} B\right) \\
\left(w, w^{\prime}\right) & \mapsto\left(w \otimes 1, w^{\prime} \otimes b\right)
\end{aligned}
$$

for some $b \in B$. It follows that there is a $k$-linear map $h: V \rightarrow B$ such that for all $w \in V$,

$$
\sigma(w)-(w \otimes 1)=v \otimes h(w) .
$$

If we continue to denote by $\sigma$ the induced coaction map $k[V] \longrightarrow k[V] \otimes_{k} B$, we see that $h$ extends to a $k[V]^{N}$-module homomorphism $k[V] \longrightarrow k[V] \otimes_{k} B$, which we continue to denote by $h$, such that for all $f \in k[V]$,

$$
\sigma(f)-(f \otimes 1)=(v \otimes 1) \cdot h(f) .
$$

We are now ready to prove the "if" direction of Theorem 1.6. Our proof is only a slight variant of the proof of the classical Chevalley-Shephard-Todd theorem presented in [Smith 1985]. 
Proof of "if" direction of Theorem 1.6. Let $R=k[V]^{G}$. By Lemma 2.6, we know that the intersection of the $k[V]^{N}$ is $R$, where $N$ runs through the pseudoreflections of $G$. By the proposition on [Smith 1985, page 225], to show $R$ is polynomial, we need only show that $k[V]$ is a free $R$-module. By graded Nakayama, the projective dimension of $k[V]$ is the smallest integer $i$ such that $\operatorname{Tor}_{i+1}^{R}(k, k[V])=0$, where $k$ is viewed as an $R$-module via the augmentation map

$$
\epsilon: k[V]^{G} \longrightarrow k[V] \longrightarrow k,
$$

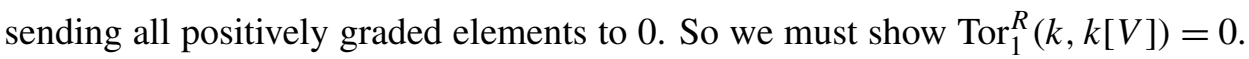

Tensoring the short exact sequence defined by $\epsilon$ with $k[V]$, we obtain a long exact sequence

$$
0 \longrightarrow \operatorname{Tor}_{1}^{R}(k, k[V]) \longrightarrow \operatorname{ker} \epsilon \otimes_{R} k[V] \stackrel{\phi}{\longrightarrow} R \otimes_{R} k[V] \stackrel{\epsilon \otimes 1}{\longrightarrow} k \otimes_{R} k[V] \longrightarrow 0 .
$$

To show $\operatorname{Tor}_{1}^{R}(k, k[V])=0$, we must prove that $\phi$ is injective. We in fact show

$$
\phi \otimes 1: \operatorname{ker} \epsilon \otimes_{R} k[V] \otimes_{k} C \longrightarrow k[V] \otimes_{k} C
$$

is injective for all finite-dimensional $k$-algebras $C$. If this is not the case, then the set

$\left\{\xi \mid C\right.$ is a finite-dimensional $k$-algebra, $\left.0 \neq \xi \in \operatorname{ker} \epsilon \otimes_{R} k[V] \otimes_{k} C,(\phi \otimes 1)(\xi)=0\right\}$ is nonempty and we can choose an element $\xi$ of minimal degree, where ker $\epsilon$ is given its natural grading as a submodule of $k[V]$ and the elements of $C$ are defined to be of degree 0 . We begin by showing $\xi \in \operatorname{ker} \epsilon \otimes_{R} R \otimes_{k} C$. That is, we show $\xi$ is fixed by all pseudoreflections.

Let $N=\operatorname{Spec} B$ be a pseudoreflection. Let $\sigma: k[V] \longrightarrow k[V] \otimes B$ be the coaction map. As explained above, we get a $k[V]^{N}$-module homomorphism $h$ : $k[V] \longrightarrow k[V] \otimes B$. Note that this morphism has degree -1 . Since

$$
(1 \otimes \sigma \otimes 1)(\xi)-\xi \otimes 1=(1 \otimes h \otimes 1)(\xi) \cdot(1 \otimes v \otimes 1 \otimes 1),
$$

the commutativity of

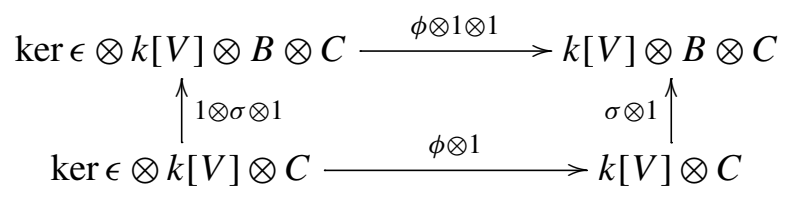

implies

$$
(\phi \otimes 1 \otimes 1)(1 \otimes h \otimes 1)(\xi) \cdot(v \otimes 1 \otimes 1)=0 .
$$

It follows that $(1 \otimes h \otimes 1)(\xi)$ is killed by $\phi \otimes 1 \otimes 1$. Since $h$ has degree -1 , our assumption on $\xi$ shows that $(1 \otimes h \otimes 1)(\xi)=0$. We therefore have $(1 \otimes \sigma \otimes 1)(\xi)=$ $\xi \otimes 1$, which proves that $\xi$ is $N$-invariant. 
Since $G$ is linearly reductive, we have a section of the inclusion $k[V]^{G} \hookrightarrow$ $k[V]$. We therefore, also obtain a section $s$ of the inclusion $j: R \hookrightarrow k[V]$. Let $\psi: \operatorname{ker} \epsilon \otimes_{R} R \longrightarrow R$ be the canonical map, and consider the diagram

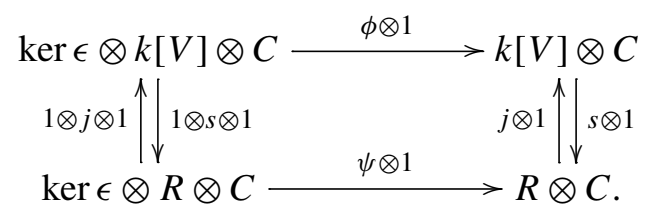

We see that

$(j \otimes 1)(\psi \otimes 1)(1 \otimes s \otimes 1)(\xi)=(\phi \otimes 1)(1 \otimes j \otimes 1)(1 \otimes s \otimes 1)(\xi)=(\phi \otimes 1)(\xi)=0$.

But $j \otimes 1$ and $\psi \otimes 1$ are injective, so $(1 \otimes s \otimes 1)(\xi)=0$. Since $\xi \in \operatorname{ker} \epsilon \otimes_{R} R \otimes_{k} C$, it follows that $\xi=0$, which is a contradiction.

Reducing the "only if" direction of Theorem 1.6 to a case of Theorem 1.9. Now that we have proved the "if" direction of Theorem 1.6, we work toward reducing the "only if" direction to the special case of Theorem 1.9 where $U=\mathbb{V}^{\vee}$. The main step in this reduction is showing that if $G$ acts faithfully on $V$, and $H$ denotes the subgroup scheme generated by pseudoreflections, then the action of $G / H$ on $\mathbb{V}^{\vee} / H$ has no pseudoreflections at the origin. In the classical case, the proof of this statement relies on the fact that $G$ has no pseudoreflections if and only if $\mathbb{V}^{\vee} \rightarrow \mathbb{V}^{\vee} / G$ is étale in codimension 1 . As the following example illustrates, this relation between pseudoreflections and ramification no longer holds in our case.

Example 2.7. Let $k$ be a field of characteristic 2 and $G=\mu_{2}$. We define an action of $G$ on $V=k x \oplus k y$ as follows: for every $k$-scheme $T$ and every section $\zeta \in G(T)$, let $\zeta$ act on $V \otimes_{k} \mathbb{O}_{T}$ by sending $x$ to $\zeta x$ and $y$ to $\zeta y$. Then $\pi: \mathbb{V}^{\vee} \rightarrow \mathbb{V}^{\vee} / G$ is a $G$-torsor away from the one singular point in $\mathbb{V}^{\vee} / G$. Hence, $\pi$ is ramified at every height 1 prime, but $G$ has no pseudoreflections.

We must therefore take a different approach to showing that the action of $G / H$ on $\mathbb{V}^{\vee} / H$ has no pseudoreflections at the origin. Our strategy is to reduce to the classical case by lifting to characteristic 0 . This is carried out after some preliminary lemmas.

Lemma 2.8. Let $G$ be a finite group scheme which acts faithfully on an affine scheme $U$. If $H$ is a normal subgroup scheme of $G$, then the action of $G / H$ on $U / H$ is faithful.

Proof. Let $\mathfrak{X}=[U / H]$ and let $\pi: U \rightarrow U / H$ be the natural map. We must show that if $G^{\prime}$ is a subgroup scheme of $G$ such that $G^{\prime} / H$ acts trivially on $U / H$, then $G^{\prime}=H$. Replacing $G$ by $G^{\prime}$, we can assume $G^{\prime}=G$. 
Since $G$ acts faithfully on $U$, there is a nonempty open substack of $\mathfrak{X}$ which is isomorphic to its coarse space. That is, we have a nonempty open subscheme $V$ of $U / H$ over which $\pi$ is an $H$-torsor. Let $P=V \times_{U / H} U$. Since $G$ acts on $P$ over $V$, we obtain a morphism

$$
s: G \longrightarrow \operatorname{Aut}(P)=H .
$$

Note that $s$ is a section of the closed immersion $H \rightarrow G$, so $H=G$.

Lemma 2.9. Let $G$ be a finite flat linearly reductive group scheme over a complete discrete valuation ring $R$ with residue field $k$. If $G$ acts linearly on $\mathbb{A}_{R}^{n}$ and $\mathbb{A}_{k}^{n} / G_{k}$ is isomorphic to $\mathbb{A}_{k}^{n}$, then $\mathbb{A}_{R}^{n} / G$ is isomorphic to $\mathbb{A}_{R}^{n}$.

Proof. Let $\mathfrak{m}$ be the maximal ideal of $R$ and let $\mathbb{A}_{R}^{n} / G=\operatorname{Spec} A$. Since $\mathbb{A}_{R}^{n}$ is flat over $R$, it follows that $\mathbb{A}_{R}^{n} / G$ is as well (see, for example, [Alper 2008, Theorem 4.16(ix)]). Since $G$ is linearly reductive,

$$
\operatorname{Spec} k \times{ }_{R} \mathbb{A}_{R}^{n} / G=\mathbb{A}_{k}^{n} / G_{k} .
$$

Choose an isomorphism

$$
\varphi_{0}: k\left[x_{1}, \ldots, x_{n}\right] \longrightarrow A \otimes_{R} k
$$

and let $r_{i} \in R$ be an arbitrary lift of $\varphi_{0}\left(x_{i}\right)$. By Nakayama's lemma, the morphism

$$
\varphi: R\left[x_{1}, \ldots, x_{n}\right] \longrightarrow A
$$

sending $x_{i}$ to $r_{i}$ is surjective. As $R$ is complete, to show $\varphi$ is an isomorphism, we need only show that the base change $\varphi_{m}$ of $\varphi$ to $R / \mathfrak{m}^{\ell+1}$ is an isomorphism for every $\ell$. This follows from the fact that $\varphi_{0}$ is an isomorphism and $A \otimes_{R} R / \mathrm{m}^{\ell}$ is flat over $R / \mathfrak{m}^{\ell}$.

Proposition 2.10. Let $G$ be a finite linearly reductive group scheme over $S$ with a faithful action on a finite-dimensional k-vector space $V$. Let $U=\mathbb{V}^{\vee}$ and let $H$ be the subgroup scheme of $G$ generated by pseudoreflections. Then the induced action of $G / H$ on $U / H \simeq \mathbb{A}_{k}^{n}$ has no pseudoreflections at the origin.

Proof. By the "if" direction of Theorem 1.6, we have $k[V]^{H}=k[W]$ for some subvector space $W$ of $k[V]$. The proof of [Neusel 2007, Proposition 6.19] shows that the degrees of the homogeneous generators of $k[V]^{H}$ are determined. As a result, the action of $G / H$ on $k[W]$ is linear. Lemma 2.8 further tells us that this action is faithful.

Assume that the subgroup scheme $H^{\prime \prime}$ of $G / H$ generated by pseudoreflections is nontrivial. Then $H^{\prime \prime}=H^{\prime} / H$ where $H^{\prime}$ is a normal subgroup scheme of $G$ which properly contains $H$. To prove $G / H$ has no pseudoreflections at the origin, it suffices by Lemma 2.3 to replace $k$ by its algebraic closure. By [Abramovich et al. 2008, Lemma 2.11], we see then that $G$ is the semidirect product of its identity 
component, which is diagonalizable, and a finite constant tame group scheme. The same is true for $H$ and $H^{\prime}$.

Let $R$ be a complete discrete valuation ring whose residue field is $k$ and whose fraction field $K$ is of characteristic 0. Lemma 2.1 shows that there exist finite flat linearly reductive group schemes $\tilde{G}, \tilde{H}$, and $\tilde{H}^{\prime}$ over $R$ whose base changes to $k$ are $G, H$, and $H^{\prime}$, respectively. Furthermore, $\tilde{H}^{\prime}$ and $\tilde{H}$ are normal closed subgroup schemes of $\tilde{G}$, and $\tilde{H}$ is a proper subgroup scheme of $\tilde{H}^{\prime}$. In characteristic 0 , every finite flat group scheme is locally constant, so after replacing $R$ by a finite extension, we can further assume that $\tilde{G}_{K}, \tilde{H}_{K}$, and $\tilde{H}_{K}^{\prime}$ are constant group schemes.

Let $\mathfrak{m}$ denote the maximal ideal of $R$ and let $R_{\ell}=R / \mathfrak{m}^{\ell}$. Let $\tilde{G}_{\ell}, \tilde{H}_{\ell}$, and $\tilde{H}_{\ell}^{\prime}$ denote the base changes of $\tilde{G}, \tilde{H}$, and $\tilde{H}^{\prime}$ to $R_{\ell}$. Choosing a basis for $V$, we can identify $U$ with $\mathbb{A}_{k}^{n}$. The $G$-action on $U$ is then given by a group scheme homomorphism $\varphi_{0}: G \longrightarrow \mathrm{GL}_{n, k}$. By [Grothendieck 1970, Exposé III 2.3], given a deformation $\varphi_{\ell}: \tilde{G}_{\ell} \longrightarrow \mathrm{GL}_{n, R_{\ell}}$ of $\varphi_{0}$, the obstruction to deforming $\varphi_{\ell}$ to a homomorphism $\varphi_{\ell+1}: \tilde{G}_{\ell+1} \longrightarrow \mathrm{GL}_{n, R_{\ell+1}}$ lies in

$$
H^{2}\left(\tilde{G}_{\ell}, \operatorname{Lie}\left(\mathrm{GL}_{n}\right) \otimes \mathfrak{m}^{\ell} / \mathfrak{m}^{\ell+1}\right),
$$

which vanishes as $\tilde{G}_{\ell}$ is linearly reductive. We therefore obtain a faithful action of $\tilde{G}$ on $\mathbb{A}_{R}^{n}$ lifting the action of $G$ on $U$.

By Lemma 2.9 , we see that $\mathbb{A}_{K}^{n} / \tilde{H}_{K}$ and $\mathbb{A}_{K}^{n} / \tilde{H}_{K}^{\prime}$ are polynomial. The classical Chevalley-Shephard-Todd theorem then shows that there is a pseudoreflection $\tilde{N}_{K}$ of $\tilde{G}_{K}$ which is contained in $\tilde{H}_{K}^{\prime}$ but not contained in $\tilde{H}_{K}$. Note that this is not yet a contradiction as it is not clear that $\tilde{H}_{K}$ is the subgroup scheme of $\tilde{G}_{K}$ generated by pseudoreflections. Let $\tilde{N}$ be the closure of $\tilde{N}_{K}$ in $\tilde{G}$. Since $\tilde{G}$ is a finite flat linearly reductive group scheme over $R$, we see that $\tilde{N}$ is as well. Since $\tilde{N}_{K}$ is a pseudoreflection, there exists some $v=\sum_{i} a_{i} x_{i} \in K\left[x_{1}, \ldots, x_{n}\right]$ such that $\tilde{N}_{K}$ acts trivially on $K\left[x_{1}, \ldots, x_{n}\right] / v$. After scaling the $a_{i}$, we can assume $a_{1} \in R^{*}$ and all $a_{i} \in R$. Consider the commutative diagram

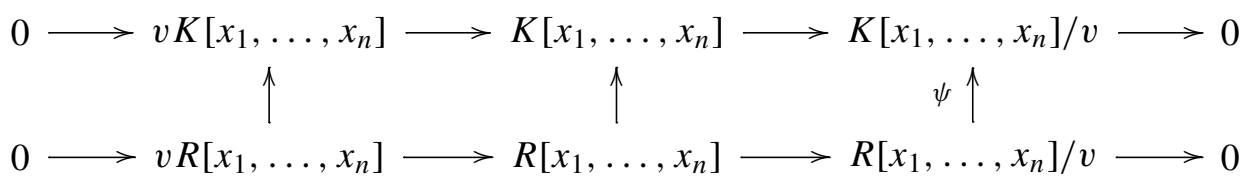

of $\tilde{N}$-comodules. Since the left square is Cartesian, we see that $\psi$ is injective. It follows that the action of $\tilde{N}$ on the hyperplane defined by $v$ in $\mathbb{A}_{R}^{n}$ is trivial. Reducing $\bmod \mathfrak{m}$, we see that $\tilde{N}_{k}$ is a pseudoreflection of $G$. Furthermore, $\tilde{N}_{k}$ is not contained in $H$, which is a contradiction.

Using Lemma 2.8 and Proposition 2.10, we now prove the "only if" direction of Theorem 1.6, assuming the special case of Theorem 1.9 in which $U=\mathbb{V}^{\vee}$. 
Proof of "only if" direction of Theorem 1.6. Let $H$ be the subgroup scheme generated by pseudoreflections. By the "if" direction, $k[V]^{H}$ is polynomial and as explained in the proof of Proposition 2.10, the $G / H$-action on $k[V]^{H}$ is linear. Since $G / H$ acts faithfully on $U / H$ without pseudoreflections at the origin by Lemma 2.8 and Proposition 2.10, and since $M=U / G$ is smooth by assumption, Theorem 1.9 implies that $U / H$ is a $G / H$-torsor over $U / G$ after potentially shrinking $U / G$. Since the origin of $U / H$ is a fixed point, we conclude that $G=H$.

\section{Theorem 1.9 for linear actions on polynomial rings}

In Section 2, we reduced the proof of the "only if" direction of Theorem 1.6 to this statement:

Proposition 3.1. Let $G$ be a stable group scheme over $S$ which acts faithfully on a finite-dimensional $k$-vector space $V$. Then Theorem 1.9 holds when $U=\mathbb{V}^{\vee}$ and $x$ is the origin.

The proof of this proposition is given in two steps. First we handle the case when $G$ is diagonalizable, and then we use that for the general case (see page 15).

Reinterpreting a result of Iwanari. The key to proving Proposition 3.1 for diagonalizable $G$ is provided by [Iwanari 2009, Theorem 3.3 and Proposition 3.4] after we reinterpret them in the language of pseudoreflections. We refer the reader to [Iwanari 2009, pages 4-6] for the basic definitions concerning monoids. We recall the following definition given as [Iwanari 2009, Definition 2.5].

Definition 3.2. An injective morphism $i: P \rightarrow F$ from a simplicially toric sharp monoid to a free monoid is called a minimal free resolution if $i$ is close and if for all injective close morphisms $i^{\prime}: P \rightarrow F^{\prime}$ to a free monoid $F^{\prime}$ of the same rank as $F$, there is a unique morphism $j: F \rightarrow F^{\prime}$ such that $i^{\prime}=j i$.

Given a faithful action of a finite diagonalizable group scheme $\Delta$ over $S$ on a $k$-vector space $V$ of dimension $n$, we can decompose $V$ as a direct sum of onedimensional $\Delta$-representations. Therefore, after choosing an appropriate basis, we have an identification of $k[V]$ with $k\left[\mathbb{N}^{n}\right]$ and can assume that the $\Delta$-action on $U=\mathbb{V}^{\vee}$ is induced from a morphism of monoids

$$
\pi: F=\mathbb{N}^{n} \longrightarrow A,
$$

where $A$ is the finite abelian group such that $\Delta$ is the Cartier dual $D(A)$ of $A$. We see then that

$$
U / \Delta=\operatorname{Spec} k[P],
$$

where $P$ is the submonoid $\{p \mid \pi(p)=0\}$ of $F$. Note that $P$ is simplicially toric sharp, that $i: P \rightarrow F$ is close, and that $A=F^{g p} / i\left(P^{g p}\right)$. 
We now give the relationship between minimal free resolutions and pseudoreflections.

Proposition 3.3. With notation as above, $i: P \rightarrow F$ is a minimal free resolution if and only if the action of $\Delta$ on $V$ has no pseudoreflections.

Proof. If $i$ is not a minimal free resolution, then without loss of generality, $i=j i^{\prime}$, where $i^{\prime}: P \rightarrow F$ is close and injective, and $j: F \rightarrow F$ is given by

$$
j\left(a_{1}, a_{2}, \ldots, a_{n}\right)=\left(m a_{1}, a_{2}, \ldots, a_{n}\right),
$$

with $m \neq 1$. We have then a short exact sequence

$$
0 \longrightarrow F^{g p} / i^{\prime}\left(P^{g p}\right) \longrightarrow F^{g p} / i\left(P^{g p}\right) \longrightarrow F^{g p} /(m, 1, \ldots, 1)\left(F^{g p}\right) \longrightarrow 0 .
$$

Let $N$ be the Cartier dual of $F^{g p} /(m, 1, \ldots, 1)\left(F^{g p}\right)$, which is a subgroup scheme of $\Delta$. Letting $\left\{x_{i}\right\}$ be the standard basis of $F$, we see that

$$
k[F]^{N}=k\left[x_{1}^{m}, x_{2}, \ldots, x_{n}\right],
$$

and so $V^{N}$, which is the degree 1 part of $k[F]^{N}$, has codimension 1 in $V$. Therefore, $N$ is a pseudoreflection.

Conversely, suppose $N$ is a pseudoreflection. Since $N$ is a subgroup scheme of $\Delta$, it is diagonalizable as well. Let $N=\operatorname{Spec} k[B]$, where $B$ is a finite abelian group, and let $\psi: A \rightarrow B$ be the induced map. We see that

$$
V^{N}=\bigoplus_{i \neq j} k x_{i}
$$

for some $j$. Without loss of generality, $j=1$. It follows then that

$$
\{f \in F \mid \psi \pi(f)=0\}=(m, 1, \ldots, 1) F
$$

for some $m$ dividing $|B|$. Since the $\Delta$ action on $V$ is assumed to be faithful, we see, in fact, that $m=|B|$. Therefore, $i$ factors through $\cdot(m, 1, \ldots, 1): F \longrightarrow F$, which shows that $i$ is not a minimal free resolution.

Having reinterpreted minimal free resolutions, the proof of Proposition 3.1 for diagonalizable group schemes $G$ follows easily from Iwanari's work.

Proposition 3.4. Let $G=\Delta$ be a finite diagonalizable group scheme over $S$ which acts faithfully on a finite-dimensional $k$-vector space $V$. Then Theorem 1.9 holds when $U=\mathbb{V}^{\vee}$ and $x$ is the origin. In this case it is not necessary to shrink $M$ to a smaller Zariski neighborhood of the image of $x$.

Proof. Let $F$ and $P$ be as above, and let $\mathfrak{X}=[U / \Delta]$. By Proposition 3.3, the morphism $i: P \rightarrow F$ is a minimal free resolution. [Iwanari 2009, Theorem 3.3 (1) and Proposition 3.4] then show that the natural morphism $\mathfrak{X} \times{ }_{M} M^{0} \rightarrow M^{0}$ is an isomorphism. Since $\mathfrak{X} \times{ }_{M} M^{0}=\left[U^{0} / \Delta\right]$, we see $U^{0}$ is a $\Delta$-torsor over $M^{0}$. 
Finishing the proof. The goal of this subsection is to prove Proposition 3.1. The main result used in the proof of this proposition, as well as in the proof of Theorem 1.9 , is the following.

Proposition 3.5. Let notation and hypotheses be as in Theorem 1.9. Let $X=U / \Delta$ and $G=\Delta \rtimes Q$, where $\Delta$ is diagonalizable and $Q$ is constant and tame. If in addition to assuming that $G$ acts without pseudoreflections at $x$, we assume that $\Delta$ is local and that the base change of $U$ to $X^{s m}$ is a $\Delta$-torsor over $X^{s m}$, then after possibly shrinking $M$ to a smaller Zariski neighborhood of the image of $x$, the quotient map $f: X \rightarrow M$ is unramified in codimension 1.

Proof. Let $g$ be the quotient map $U \rightarrow X$. For every $q \in Q$, consider the Cartesian diagram

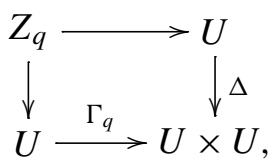

where $\Gamma_{q}(u)=(u, q u)$. We see that $Z_{q}$ is a closed subscheme of $U$ and that $Z_{q}(T)$ is the set of $u \in U(T)$ which are fixed by $q$. Let $Z$ be the closed subset of $U$ which is the union of the $Z_{q}$ for $q \neq 1$. Since the action of $G$ on $U$ is faithful, $Z$ is not all of $U$. Let $Z^{\prime}$ be the union of the codimension 1 components of $Z$. Since $f g$ is finite, we see that $f g\left(Z^{\prime}\right)$ is a closed subset of $M$. Moreover, $f g\left(Z^{\prime}\right)$ does not contain the image of $x$, as $G$ is assumed to act without pseudoreflections at $x$. By shrinking $M$ to $M-f g\left(Z^{\prime}\right)$, we can assume that no nontrivial $q \in Q$ acts trivially on a divisor of $U$.

Let $U=\operatorname{Spec} R$. The morphism $f$ is unramified in codimension 1 if and only if the (traditional) inertia groups of all height 1 primes $\mathfrak{p}$ of $R^{\Delta}$ are trivial. So, we must show that if $q \in Q$ acts trivially on $V(\mathfrak{p})$, then $q=1$. Since $g$ is finite, and hence integral, the going-up theorem shows that

$$
\mathfrak{p} R=\mathfrak{P}_{1}^{e_{1}}+\cdots+\mathfrak{P}_{n}^{e_{n}},
$$

where the $\mathfrak{P}_{i}$ are height 1 primes and the $e_{i}$ are positive integers. Note that $X$ is normal and so the complement of $X^{s m}$ in $X$ has codimension at least 2. As a result,

$$
h: U \times{ }_{X} \operatorname{Spec} \mathrm{O}_{X, \mathfrak{p}} \longrightarrow \operatorname{Spec} \mathrm{O}_{X, \mathfrak{p}}
$$

is a $\Delta$-torsor. Since $\Delta$ is local, $h$ is a homeomorphism of topological spaces, so there is exactly one prime $\mathfrak{P}$ lying over $\mathfrak{p}$. We see then that $U \times_{X} V(\mathfrak{p})=V\left(\mathfrak{P}^{e}\right)$ for some $e$.

Let $V(\mathfrak{p})^{0}$ be the intersection of $V(\mathfrak{p})$ with $X^{s m}$, and let $Z^{0}=U \times_{X} V(\mathfrak{p})^{0}$. Then $Z^{0}$ is a $\Delta$-torsor over $V(\mathfrak{p})^{0}$. Since $q$ acts trivially on $V(\mathfrak{p})$, we obtain an action of 
$q$ on $Z^{0}$ over $V(\mathfrak{p})^{0}$, and hence a group scheme homomorphism

$$
\varphi: Q_{V(\mathfrak{p})^{0}}^{\prime} \longrightarrow \operatorname{Aut}\left(Z^{0} / V(\mathfrak{p})^{0}\right)=\Delta_{V(\mathfrak{p})^{0}},
$$

where $Q^{\prime}$ denotes the subgroup of $Q$ generated by $q$. Since $V(\mathfrak{p})^{0}$ is reduced, we see that $\varphi$ factors through the reduction of $\Delta_{V(\mathfrak{p})^{0}}$, which is the trivial group scheme. Therefore, $q$ acts trivially on $Z^{0}$.

Since the complement of $X^{s m}$ in $X$ has codimension at least 2, and since $g$ factors as a flat map $U \rightarrow[U / \Delta]$ followed by a coarse space map $[U / \Delta] \rightarrow X$, both of which are codimension-preserving (see [Fantechi et al. 2007, Definition 4.2 and Remark 4.3]), we see that the complement of $Z^{0}$ in $V\left(\mathfrak{P}^{e}\right)$ has codimension at least 2. Note that if $Y$ is a normal scheme and $W$ is an open subscheme of $Y$ whose complement has codimension at least 2, then any morphism from $W$ to an affine scheme $Z$ extends uniquely to a morphism from $Y$ to $Z$. Since the action of $q$ on $V\left(\mathfrak{P}^{e}\right)$ restricts to a trivial action on $Z^{0}$, the action of $q$ on $V\left(\mathfrak{P}^{e}\right)$ is trivial. Therefore, $q$ acts trivially on a divisor of $U$, and so $q=1$.

Proof of Proposition 3.1. Let $k^{\prime} / k$ be a finite Galois extension such that $G_{k^{\prime}} \simeq$ $\Delta \rtimes Q$, where $\Delta$ is diagonalizable and $Q$ is constant and tame. Let $S^{\prime}=\operatorname{Spec} k^{\prime}$ and consider the diagram

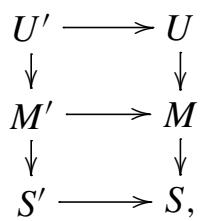

where the squares are Cartesian. We denote by $x^{\prime}$ the induced $k^{\prime}$-rational point of $U^{\prime}$. Since $\Delta$ is the product of a local diagonalizable group scheme and a locally constant diagonalizable group scheme, replacing $k^{\prime}$ by a further extension if necessary, we can assume that $\Delta$ is local.

Since $G$ is stable, $G_{k^{\prime}}$ has no pseudoreflections at $x^{\prime}$. It follows then from Proposition 3.5 that there exists an open neighborhood $W^{\prime}$ of $x^{\prime}$ such that $U^{\prime} \times{ }_{M^{\prime}}$ $W^{\prime} \longrightarrow W^{\prime}$ is unramified in codimension 1 . Since $k^{\prime} / k$ is a finite Galois extension, replacing $W^{\prime}$ by the intersection of the $\tau\left(W^{\prime}\right)$ as $\tau$ ranges over the elements of $\operatorname{Gal}\left(k^{\prime} / k\right)$, we can assume $W^{\prime}$ is Galois-invariant. Hence, $W^{\prime}=W \times_{M} M^{\prime}$ for some open subset $W$ of $M$. We shrink $M$ to $W$.

To check that $U^{0}$ is a $G$-torsor over $M^{0}$, we can look étale-locally. We can therefore assume $S=S^{\prime}$. Let $X=U / \Delta$, and let $g: U \rightarrow X$ and $f: X \rightarrow M$ be the quotient maps. We denote by $X^{0}$ the fiber product $X \times_{M} M^{0}$ and by $f^{0}$ the induced morphism $X^{0} \rightarrow M^{0}$.

By Proposition 3.4, we know that the base change of $U$ to $X^{s m}$ is a $\Delta$-torsor over $X^{s m}$. Since $f$ is unramified in codimension 1 , we see that $f^{0}$ is as well. Since $M^{0}$ 
is smooth and $X^{0}$ is normal, the purity of the branch locus theorem [Grothendieck and Raynaud 1971, X.3.1] implies that $f^{0}$ is étale, and hence a $Q$-torsor. Since $X^{0}$ is étale over $M^{0}$, it is smooth. As a result, $U^{0}$ is a $\Delta$-torsor over $X^{0}$ from which it follows that $U^{0}$ is a $G$-torsor over $M^{0}$.

This finishes the proof of Proposition 3.1, and hence also of Theorem 1.6. We conclude this section by proving Corollary 1.8 .

Proof of Corollary 1.8. Let $U=\operatorname{Spec} R$ and $M=U / G$. We denote by $y$ the image of $x$. Since $G$ being generated by pseudoreflections at $x$ implies that $G_{K}$ is generated by pseudoreflections at $x$ for arbitrary finite linearly reductive group schemes $G$, and since smoothness of $M$ at $y$ can be checked étale-locally, we can assume that $x$ is $k$-rational. Let $V=\mathfrak{m}_{x} / \mathfrak{m}_{x}^{2}$ be the cotangent space of $x$. As $G$ is linearly reductive, there is a $G$-equivariant section of $\mathfrak{m}_{x} \rightarrow V$. This yields a $G$-equivariant map $\operatorname{Sym}^{\bullet}(V) \rightarrow R$, which induces an isomorphism $k \llbracket V \rrbracket \longrightarrow \hat{\mathrm{O}}_{U, x}$ of $G$-representations. That is, complete locally, we have linearized the $G$-action. Since $\hat{\widehat{O}}_{M, y}=k[[V]]^{G}$, the corollary follows from Theorem 1.6, as $M$ is smooth at $y$ if and only if $\hat{\mathcal{O}}_{M, y}$ is a formal power series ring over $k$.

\section{Actions on smooth schemes}

Having proved Theorem 1.9 for polynomial rings with linear actions, we now turn to the general case. We begin with two preliminary lemmas and a technical proposition.

Lemma 4.1. Let $U$ be a smooth affine scheme over $S$ with an action of a finite diagonalizable group scheme $\Delta$. Then there is a closed subscheme $Z$ of $U$ on which $\Delta$ acts trivially, with the property that every closed subscheme $Y$ on which $\Delta$ acts trivially factors through $Z$. Furthermore, the construction of $Z$ commutes with flat base change on $U / \Delta$.

Proof. Let $U=\operatorname{Spec} R$ and $\Delta=\operatorname{Spec} k[A]$, where $A$ is a finite abelian group written additively. The $\Delta$-action on $U$ yields an $A$-grading

$$
R=\bigoplus_{a \in A} R_{a}
$$

We see that if $\mathscr{F}$ is an ideal of $R$, then $\Delta$ acts trivially on $Y=\operatorname{Spec} R / \mathscr{F}$ if and only if $\mathscr{g}$ contains the $R_{a}$ for $a \neq 0$. Letting $\Phi$ be the ideal generated by the $R_{a}$ for $a \neq 0$, we see that Spec $R / \mathscr{I}$ is our desired $Z$.

We now show that the formation of $Z$ commutes with flat base change. Note that

$$
U / \Delta=\operatorname{Spec} R_{0}
$$


Let $R_{0}^{\prime}$ be a flat $R_{0}$-algebra and let $R^{\prime}=R_{0}^{\prime} \otimes_{R_{0}} R$. The induced $\Delta$-action on Spec $R^{\prime}$ corresponds to the $A$-grading

$$
R^{\prime}=\bigoplus_{a \in A}\left(R_{0}^{\prime} \otimes_{R_{0}} R_{a}\right)
$$

Since $R_{0}^{\prime}$ is flat over $R_{0}$, we see that $\mathscr{I} \otimes_{R_{0}} R_{0}^{\prime}$ is an ideal of $R^{\prime}$, and one easily shows that it is the ideal generated by the $R_{0}^{\prime} \otimes_{R_{0}} R_{a}$ for $a \neq 0$.

Recall that if $G$ is a group scheme over a base scheme $B$ which acts on a $B$ scheme $U$, and if $y: T \rightarrow U$ is a morphism of $B$-schemes, then the stabilizer group scheme $G_{y}$ is defined by the Cartesian diagram

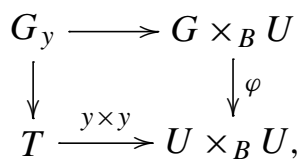

where $\varphi(g, u)=(g u, u)$. If $U$ is separated over $B$, then $G_{y}$ is a closed subgroup scheme of $G_{T}$.

Lemma 4.2. Let $B$ be a scheme and $G$ a finite flat group scheme over B. If $G$ acts on a $B$-scheme $U$, then $U \rightarrow U / G$ is a $G$-torsor if and only if the stabilizer group schemes $G_{y}$ are trivial for all closed points $y$ of $U$.

Proof. The "only if" direction is clear. To prove the "if" direction, it suffices to show that the stabilizer group schemes $G_{y}$ are trivial for all scheme-valued points $y: T \rightarrow U$. This is equivalent to showing that the universal stabilizer $G_{u}$ is trivial, where $u: U \rightarrow U$ is the identity map. Since $G_{u}$ is a finite group scheme over $U$, it is given by a coherent sheaf $\mathscr{F}$ on $U$. The support of $\mathscr{F}$ is a closed subset, and so to prove $G_{u}$ is trivial, it suffices to check this on stalks of closed points. Nakayama's lemma then shows that we need only check the triviality of $G_{u}$ on closed fibers. That is, we need only check that the $G_{y}$ are trivial for closed points $y$ of $U$.

Proposition 4.3. Let $U$ be a smooth affine scheme over $S$ with a faithful action of a stable group scheme $G$ fixing a k-rational point $x$. If $N$ has a pseudoreflection at $x$, then there is an étale neighborhood $T \longrightarrow U / G$ of $x$ and a divisor $D$ of $U_{T}$ defined by a principal ideal on which $N_{T}$ acts trivially.

Proof. Let $M=U / G$ and let $y$ be the image of $x$ in $M$. As in the proof of Corollary 1.8 , we have an isomorphism $k[[V]] \longrightarrow \hat{O}_{U, x}$ of $G$-representations, where $V=\mathfrak{m}_{x} / \mathfrak{m}_{x}^{2}$. If $N$ is a pseudoreflection at $x$, then there is some $v \in V$ such that $N$ acts trivially on the closed subscheme of Spec $k \llbracket V \rrbracket$ defined by the prime ideal generated by $v$.

Consider the contravariant functor $F$ which sends an $M$-scheme $T$ to the set of divisors of $U_{T}$ defined by a principal ideal on which $N_{T}$ acts trivially. As $F$ is 
locally of finite presentation and $U \times_{M}$ Spec $\hat{O}_{M, y}=\operatorname{Spec} \hat{O}_{U, x}$, Artin's approximation theorem [Artin 1969] finishes the proof.

We are now ready to prove Theorem 1.9. Our method of proof is similar to that of Proposition 3.1; we first prove the theorem in the case that $G$ is diagonalizable and then make use of this case to prove the theorem in general.

Proposition 4.4. Theorem 1.9 holds when $G=\Delta$ is a finite diagonalizable group scheme.

Proof. Let $g: U \rightarrow M$ be the quotient map. Since any subgroup scheme $N$ of $\Delta$ is again finite diagonalizable, Lemma 4.1 shows that for every $N$, there exists a closed subscheme $Z_{N}$ of $U$ on which $N$ acts trivially, with the property that every closed subscheme $Y$ on which $N$ acts trivially factors through $Z_{N}$. Let $Z$ be the union of the finitely many closed subsets $Z_{N}$ for $N \neq 1$. Since the action of $\Delta$ on $U$ is faithful, $Z$ has codimension at least 1 . Let $Z^{\prime}$ be the union of all irreducible components of $Z$ which have codimension 1 . Since $\Delta$ acts without pseudoreflections at $x$, we see $x \notin Z^{\prime}$. Note that $g\left(Z^{\prime}\right)$ is closed as $g$ is proper. Since the construction of $Z$ commutes with flat base change on $M$ and since flat morphisms are codimension-preserving, replacing $M$ with $M-g\left(Z^{\prime}\right)$, we can assume that there are no nontrivial subgroup schemes of $\Delta$ which fppf locally on $M$ act trivially on a divisor of $U$.

By Lemma 4.2, to show $U^{0}$ is a $\Delta$-torsor over $M^{0}$, it suffices to show that for every closed point $y$ of $U$ which maps to $M^{0}$, the stabilizer group scheme $\Delta_{y}$ is trivial. Fix such a closed point $y$ and let $T=\operatorname{Spec} k(y)$. Since $T$ is fppf over $S$, we see from Proposition 4.3 that the closed subgroup scheme $\Delta_{y}$ of $\Delta_{T}$ acts faithfully on $U_{T}$ without pseudoreflections at the $k(y)$-rational point $y^{\prime}$ of $U_{T}$ induced by $y$. Since $y$ maps to a smooth point of $M$, it follows that $y^{\prime}$ maps to a smooth point of $M_{T}$. Corollary 1.8 then shows that $\Delta_{y}$ is generated by pseudoreflections. Since $\Delta_{y}$ has no pseudoreflections, it is therefore trivial.

Proof of Theorem 1.9. If $G=\Delta \rtimes Q$, where $\Delta$ is diagonalizable and $Q$ is constant and tame, then letting $Z^{\prime}$ be as in Proposition 4.4 and letting $U, X, f$, and $g$ be as in the proof of Proposition 3.1, the proof of Proposition 4.4 shows that after replacing $M$ by $M-f g\left(Z^{\prime}\right)$, the base change of $U$ to $X^{s m}$ is a $\Delta$-torsor over $X^{s m}$. As in the proof of Proposition 3.1, we can then reduce the general case to the case when $G=\Delta \rtimes Q$, where $\Delta$ is local diagonalizable and $Q$ is constant tame. The last paragraph of the proof of Proposition 3.1 then shows that $U^{0}$ is a $G$-torsor over $M^{0}$.

\section{Schemes with linearly reductive singularities}

Let $k$ be a perfect field of characteristic $p$. 
Definition 5.1. We say a scheme $M$ over $S$ has linearly reductive singularities if there is an étale cover $\left\{U_{i} / G_{i} \rightarrow M\right\}$, where the $U_{i}$ are smooth over $S$ and the $G_{i}$ are linearly reductive group schemes which are finite over $S$.

Note that if $M$ has linearly reductive singularities, then it is automatically normal and, in fact, Cohen-Macaulay by [Hochster and Roberts 1974, page 115].

Our goal in this section is to prove Theorem 1.10, which generalizes the result that every scheme with quotient singularities prime to the characteristic is the coarse space of a smooth Deligne-Mumford stack. We remark that in the case of quotient singularities, the converse of the analogous theorem is true as well; that is, every scheme which is the coarse space of a smooth Deligne-Mumford stack has quotient singularities. It is not clear, however, that the converse of Theorem 1.10 should hold. We know from [Abramovich et al. 2008, Theorem 3.2] that $\mathfrak{X}$ is étale-locally $\left[V / G_{0}\right]$, where $G_{0}$ is a finite flat linearly reductive group scheme over $V / G_{0}$, but $V$ need not be smooth and $G_{0}$ need not be the base change of a group scheme over $S$. On the other hand, Proposition 5.2 below shows that $\mathfrak{X}$ is étale-locally $[U / G]$ where $U$ is smooth and $G$ is a group scheme over $S$, but here $G$ is not finite.

Before proving Theorem 1.10, we begin with a technical proposition followed by a series of lemmas.

Proposition 5.2. Let $\mathfrak{X}$ be a tame stack over $S$ with coarse space $M$. Then there exists an étale cover $T \rightarrow M$ such that

$$
\mathfrak{X} \times{ }_{M} T=\left[U / \mathbb{G}_{m, T}^{r} \rtimes H\right],
$$

where $H$ is a finite constant tame group scheme and $U$ is affine over T. Furthermore, $\mathbb{G}_{m, T}^{r} \rtimes H$ is the base change to $T$ of a group scheme $\mathbb{G}_{m, S}^{r} \rtimes H$ over $S$, so $\mathfrak{X} \times{ }_{M} T=\left[U / \mathbb{G}_{m, S}^{r} \rtimes H\right]$.

Proof. [Abramovich et al. 2008, Theorem 3.2] shows that there exists an étale cover $T \rightarrow M$ and a finite flat linearly reductive group scheme $G_{0}$ over $T$ acting on a finite finitely presented scheme $V$ over $T$ such that

$$
\mathfrak{X} \times{ }_{M} T=\left[V / G_{0}\right] .
$$

By [Abramovich et al. 2008, Lemma 2.20], after replacing $T$ by a finer étale cover if necessary, we can assume there is a short exact sequence

$$
1 \longrightarrow \Delta \longrightarrow G_{0} \longrightarrow H \longrightarrow 1
$$

where $\Delta=\operatorname{Spec}_{T}[A]$ is a finite diagonalizable group scheme and $H$ is a finite constant tame group scheme. Since $\Delta$ is abelian, the conjugation action of $G_{0}$ on $\Delta$ passes to an action

$$
H \longrightarrow \operatorname{Aut}(\Delta)=\operatorname{Aut}(A) .
$$


Choosing a surjection $F \rightarrow A$ in the category of $\mathbb{Z}[H]$-modules from a free module $F$ yields an $H$-equivariant morphism $\Delta \hookrightarrow \mathbb{G}_{m, T}^{r}$. Using the $H$-action on $\mathbb{G}_{m, T}^{r}$, we define the group scheme $\mathbb{G}_{m, T}^{r} \rtimes G_{0}$ over $T$. Note that there is an embedding

$$
\Delta \hookrightarrow \mathbb{G}_{m, T}^{r} \rtimes G_{0}
$$

sending $\delta$ to $\left(\delta, \delta^{-1}\right)$, which realizes $\Delta$ as a normal subgroup scheme of $\mathbb{G}_{m, T}^{r} \rtimes G_{0}$. We can therefore define

$$
G:=\left(\mathbb{G}_{m, T}^{r} \rtimes G_{0}\right) / \Delta .
$$

One checks that there is a commutative diagram

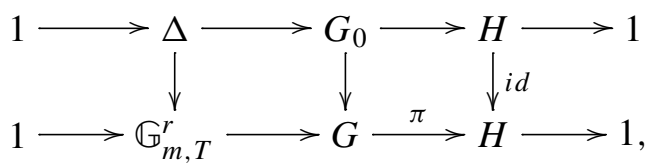

where the rows are exact and the vertical arrows are injective.

We show that, étale-locally on $T$, there is a group scheme-theoretic section of $\pi$, so that $G=\mathbb{G}_{m, T}^{r} \rtimes H$. Let $P$ be the sheaf on $T$ such that for any $T$-scheme $W, P(W)$ is the set of group scheme-theoretic sections of $\pi_{W}: G_{W} \rightarrow H_{W}$. Note that the sheaf $\operatorname{Hom}(H, G)$ parametrizing group scheme homomorphisms from $H$ to $G$ is representable since it is a closed subscheme of $G^{\times|H|}$ cut out by suitable equations. We see that $P$ is the equalizer of the two maps

$$
\underline{\operatorname{Hom}}(H, G) \underset{p_{2}}{\stackrel{p_{1}}{\longrightarrow}} H^{\times|H|},
$$

where $p_{1}(\phi)=(\pi \phi(h))_{h}$ and $p_{2}(\phi)=(h)_{h}$. That is, there is a Cartesian diagram

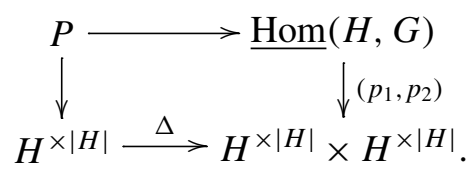

Since $H$ is separated over $T$, we see that $P$ is a closed subscheme of $\underline{\operatorname{Hom}}(H, G)$. In particular, it is representable and locally of finite presentation over $T$. Furthermore, $P \rightarrow T$ is surjective as [Abramovich et al. 2008, Lemma 2.16] shows that it has a section fppf locally. To show $P$ has a section étale locally, by [Grothendieck 1967, 17.16.3], it suffices to prove $P$ is smooth over $T$.

Given a commutative diagram

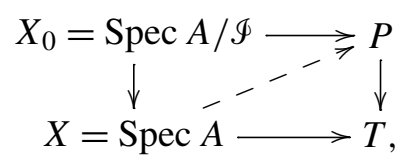


with $\mathscr{I}$ a square zero ideal, we want to find a dotted arrow making the diagram commute. That is, given a group scheme-theoretic section $s_{0}: G_{W_{0}} \rightarrow H_{W_{0}}$ of $\pi_{W_{0}}$, we want to find a group scheme homomorphism $s: G_{W} \rightarrow H_{W}$ which pulls back to $s_{0}$ such that $\pi_{W} \circ S$ is the identity. Note first that any group scheme homomorphism $s$ which pulls back to $s_{0}$ is automatically a section of $\pi_{W}$ since $H$ is a finite constant group scheme and $\pi_{W} \circ s$ pulls back to the identity over $W_{0}$. By [Grothendieck 1970, Exposé III 2.3], the obstruction to lifting $s_{0}$ to a group scheme homomorphism lies in

$$
H^{2}(H, \operatorname{Lie}(G) \otimes \mathscr{I})
$$

which vanishes as $H$ is linearly reductive. This proves the smoothness of $P$.

To complete the proof of the lemma, let $U:=V \times{ }^{G_{0}} G$ and note that

$$
\mathfrak{X} \times{ }_{M} T=\left[V / G_{0}\right]=[U / G] .
$$

Since $V$ is finite over $T$ and $G$ is affine over $T$, it follows that $U$ is affine over $T$ as well. Replacing $T$ by a finer étale cover if necessary, we have

$$
\mathfrak{X} \times{ }_{M} T=\left[U / \mathbb{G}_{m, T}^{r} \rtimes H\right] .
$$

Lastly, the scheme underlying $\mathbb{G}_{m, T}^{r} \rtimes H$ is $\mathbb{G}_{m, T}^{r} \times_{T} H$ and its group scheme structure is determined by the action $H \rightarrow \operatorname{Aut}\left(\mathbb{G}_{m, T}^{r}\right)$. Since $\operatorname{Aut}\left(\mathbb{G}_{m, T}^{r}\right)=\operatorname{Aut}\left(\mathbb{Z}^{r}\right)$, we can use this same action to define the semidirect product $\mathbb{G}_{m, S}^{r} \rtimes H$ and it is clear that this group scheme base changes to $\mathbb{G}_{m, T}^{r} \rtimes H$.

Lemma 5.3. If $V$ is a smooth $S$-scheme with an action of finite linearly reductive group scheme $G_{0}$ over $S$, then $\left[V / G_{0}\right]$ is smooth over $S$.

Proof. Let $\mathfrak{X}=\left[V / G_{0}\right]$. To prove $\mathfrak{X}$ is smooth, it suffices to work étale-locally on $S$, where, by [Abramovich et al. 2008, Lemma 2.20], we can assume $G_{0}$ fits into a short exact sequence

$$
1 \longrightarrow \Delta \longrightarrow G_{0} \longrightarrow H \longrightarrow 1
$$

where $\Delta$ is a finite diagonalizable group scheme and $H$ is a finite constant tame group scheme. Let $G$ be obtained from $G_{0}$ as in the proof of Proposition 5.2 and let $U=V \times{ }^{G_{0}} G$. Since $\mathfrak{X}=[U / G]$, it suffices to show $U$ is smooth over $S$. The action of $G_{0}$ on $V \times G$, given by $g_{0} \cdot(v, g)=\left(v g_{0}, g_{0} g\right)$, is free as the $G_{0}$-action on $G$ is free. As a result, $U=\left[(V \times G) / G_{0}\right]$ and $G / G_{0}=\left[G / G_{0}\right]$. Since the projection map $p: V \times G \rightarrow G$ is $G_{0}$-equivariant, we have a Cartesian diagram

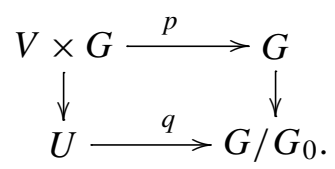


Since $p$ is smooth, $q$ is as well. Since $G \rightarrow\left[G / G_{0}\right]=G / G_{0}$ is flat and $G$ is smooth, [Grothendieck 1967, 17.7.7] shows that $G / G_{0}$ is smooth, and so $U$ is as well.

Lemma 5.4. Let $X$ be a smooth $S$-scheme and $i: U \hookrightarrow X$ an open subscheme whose complement has codimension at least 2 . Let $P$ be a $G$-torsor on $U$, where $G=\mathbb{G}_{m}^{r} \rtimes H$ and $H$ is a finite constant étale group scheme. Then $P$ extends uniquely to a $G$-torsor on $X$.

Proof. The structure map from $P$ to $U$ factors as $P \rightarrow P_{0} \rightarrow U$, where $P$ is a $\mathbb{G}_{m}^{r}$-torsor over $P_{0}$ and $P_{0}$ is an $H$-torsor over $U$. Since the complement of $U$ in $X$ has codimension at least 2, we have $\pi_{1}(U)=\pi_{1}(X)$ and so $P_{0}$ extends uniquely to an $H$-torsor $Q_{0}$ on $X$. Let $i_{0}: P_{0} \hookrightarrow Q_{0}$ be the inclusion map. Since $Q_{0}$ is smooth and the complement of $P_{0}$ in $Q_{0}$ has codimension at least 2, the natural $\operatorname{map} \operatorname{Pic}\left(Q_{0}\right) \rightarrow \operatorname{Pic}\left(P_{0}\right)$ is an isomorphism. It follows that any line bundle over $P_{0}$ can be extended uniquely to a line bundle over $Q_{0}$. We can therefore inductively construct a unique lift of $P$ over $X$.

Our proof of the following lemma closely follows that of [Fantechi et al. 2007, Theorem 4.6].

Lemma 5.5. Let $f:$ y $\rightarrow M$ be an $S$-morphism from a smooth tame stack y to its coarse space which pulls back to an isomorphism over the smooth locus $M^{0}$ of $M$. If $h: \mathfrak{X} \rightarrow M$ is a dominant, codimension-preserving morphism (see [Fantechi et al. 2007, Definition 4.2]) from a smooth tame stack, then there is a morphism $g: \mathfrak{X} \rightarrow \mathscr{Y}$, unique up to unique isomorphism, such that $f g=h$.

Proof. We show that if such a morphism $g$ exists, then it is unique. Suppose $g_{1}$ and $g_{2}$ are two such morphisms. We see then that $\left.g_{1}\right|_{h^{-1}\left(M^{0}\right)}=\left.g_{2}\right|_{h^{-1}\left(M^{0}\right)}$. Since $h$ is dominant and codimension-preserving, $h^{-1}\left(M^{0}\right)$ is open and dense in $\mathfrak{X}$. [Fantechi et al. 2007, Proposition 1.2] shows that if $\mathfrak{X}$ and $\mathscr{Y}$ are Deligne-Mumford with $\mathfrak{X}$ normal and $\mathscr{Y}$ separated, then $g_{1}$ and $g_{2}$ are uniquely isomorphic. The proof, however, applies equally well to tame stacks since the only key ingredient used about Deligne-Mumford stacks is that they are locally $[U / G]$ where $G$ is a separated group scheme.

By uniqueness, to show the existence of $g$, we can assume by Proposition 5.2 that $\mathscr{y}=[U / G]$, where $U$ is smooth and affine, and $G=\mathbb{G}_{m}^{r} \rtimes H$, where $H$ is a finite constant tame group scheme. Let $p: V \rightarrow \mathfrak{X}$ be a smooth cover by a smooth scheme. Since smooth morphisms are dominant and codimension-preserving, uniqueness implies that to show the existence of $g$, we need only show there is a morphism $g_{1}: V \rightarrow$ Y such that $f g_{1}=h p$. So, we can assume $\mathfrak{X}=V$.

Given a stack $\mathscr{L}$ over $M$, let $\mathscr{L}^{0}=M^{0} \times_{M} \mathscr{L}$. Given a morphism $\pi: \mathscr{L}_{1} \rightarrow \mathscr{L}_{2}$ of $M$-stacks, let $\pi^{0}: \mathscr{E}_{1}^{0} \rightarrow \mathscr{E}_{2}^{0}$ denote the induced morphism. Since $f^{0}$ is an 
isomorphism, there is a morphism $g^{0}: V^{0} \rightarrow \mathrm{y}^{0}$ such that $f^{0} g^{0}=h^{0}$. It follows that there is a $G$-torsor $P^{0}$ over $V^{0}$ and a $G$-equivariant map from $P^{0}$ to $U^{0}$ such that the diagram

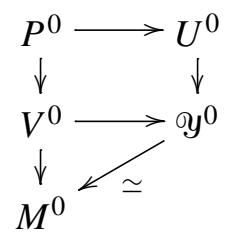

commutes and the square is Cartesian. By Lemma 5.4, $P^{0}$ extends to a $G$-torsor $P$ over $V$.

Note that if $X$ is a normal algebraic space and $i: W \hookrightarrow X$ is an open subalgebraic space whose complement has codimension at least 2 , then any morphism from $W$ to an affine scheme $Y$ extends uniquely to a morphism $X \rightarrow Y$. As a result, the morphism from $P^{0}$ to $U^{0}$ extends to a morphism $q: P \rightarrow U$. Consider the diagram

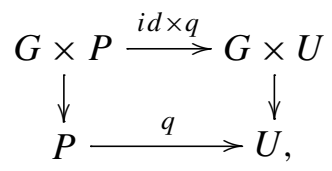

where the vertical arrows are the action maps. Precomposing either of the two maps in the diagram from $G \times P$ to $U$ by the inclusion $G \times P^{0} \hookrightarrow G \times P$ yields the same morphism. That is, the two maps from $G \times P$ to $U$ are both extensions of the same map from $G \times P^{0}$ to the affine scheme $U$, and hence are equal. This shows that $q$ is $G$-equivariant, and therefore yields a map $g: V \rightarrow$ Yy such that $f g=h$.

Proof of Theorem 1.10. We begin with the following observation. Suppose $U$ is smooth and affine over $S$ with a faithful action of a finite linearly reductive group scheme $G$ over $S$. Let $y$ be a closed point of $U$ mapping to $x \in U / G$. After making the étale base change $\operatorname{Spec} k(y) \rightarrow S$, we can assume $y$ is a $k$-rational point. Let $G_{y}$ be the stabilizer subgroup scheme of $G$ fixing $y$. Since

$$
U / G_{y} \longrightarrow U / G
$$

is étale at $y$, replacing $U / G$ by an étale cover, we can further assume that $G$ fixes $y$. Then by Corollary 1.8 , we can assume $G$ has no pseudoreflections at $y$, and, hence, Theorem 1.9 shows that after shrinking $U / G$ about $x$, we can assume that the base change of $U$ to the smooth locus of $U / G$ is a $G$-torsor.

We now turn to the proof. Since $M$ has linearly reductive singularities, there is an étale cover $\left\{U_{i} / G_{i} \rightarrow M\right\}$, where $U_{i}$ is smooth and affine over $S$ and $G_{i}$ is a finite linearly reductive group scheme over $S$ which acts faithfully on $U_{i}$. By the above discussion, replacing this étale cover by a finer étale cover if necessary, we can 
assume that the base change of $U_{i}$ to the smooth locus of $U_{i} / G_{i}$ is a $G_{i}$-torsor. Let $M_{i}=U_{i} / G_{i}$ and $\mathfrak{X}_{i}=\left[U_{i} / G_{i}\right]$. We see that the $\mathfrak{X}_{i}$ are locally the desired stacks, so we need only glue the $\mathfrak{X}_{i}$. Let $M_{i j}=M_{i} \times_{M} M_{j}$ and let $V_{i} \rightarrow \mathfrak{X}_{i}$ be a smooth cover. Since $M_{i j}$ is the coarse space of both $\mathfrak{X}_{i} \times_{M_{i}} M_{i j}$ and $\mathfrak{X}_{j} \times_{M_{j}} M_{i j}$, and since coarse space maps are dominant and codimension-preserving, Lemma 5.5 shows that there is a unique isomorphism of $\mathfrak{X}_{i} \times{ }_{M_{i}} M_{i j}$ and $\mathfrak{X}_{j} \times_{M_{j}} M_{i j}$. Identifying these two stacks via this isomorphism, let $I_{i j}$ be the fiber product over the stack of $V_{i} \times{ }_{M_{i}} M_{i j}$ and $V_{j} \times{ }_{M_{j}} M_{i j}$. We see then that we have a morphism $I_{i j} \rightarrow U_{i} \times{ }_{M} U_{j}$. This yields a groupoid

$$
\coprod I_{i j} \longrightarrow \coprod U_{i} \times{ }_{M} U_{j},
$$

which defines our desired glued stack $\mathfrak{X}$. Note that $\mathfrak{X}$ is smooth and tame by [Abramovich et al. 2008, Theorem 3.2].

\section{Acknowledgments}

I would like to thank Dustin Cartwright, Ishai Dan-Cohen, Anton Geraschenko, and David Rydh for many helpful conversations. I am of course indebted to my advisor, Martin Olsson, for his suggestions and help in editing this paper. I thank Dan Edidin for suggesting that I write up these results in this stand-alone paper rather than include them in [Satriano 2009]. Lastly, I would like to thank the referee for his helpful comments.

\section{References}

[Abramovich et al. 2008] D. Abramovich, M. Olsson, and A. Vistoli, "Tame stacks in positive characteristic", Ann. Inst. Fourier (Grenoble) 58 (2008), 1057-1091. MR 2009c:14002 Zbl 1222.14004 [Alper 2008] J. Alper, Good moduli spaces for Artin stacks, Ph.D thesis, Stanford University, 2008, Available at http://tinyurl.com/7kfaokm. MR 2711743 arXiv 0804.2242v3

[Artin 1969] M. Artin, "Algebraic approximation of structures over complete local rings", Inst. Hautes Études Sci. Publ. Math. 36 (1969), 23-58. MR 42 \#3087 Zbl 0181.48802

[Bourbaki 1968] N. Bourbaki, Éléments de mathématique. Fasc. XXXIV, Actualités Scientifiques et Industrielles 1337, Hermann, Paris, 1968. MR 39 \#1590

[Conrad 2004] B. Conrad, "The Keel-Mori theorem via stacks", unpublished notes, 2004, Available at http://math.stanford.edu/ conrad/papers/coarsespace.pdf.

[Fantechi et al. 2007] B. Fantechi, E. Mann, and F. Nironi, "Smooth toric DM stacks", preprint, 2007. arXiv $0708.1254 \mathrm{v} 1$

[Grothendieck 1967] A. Grothendieck, "Éléments de géométrie algébrique, IV: Étude locale des schémas et des morphismes de schémas, IV”, Inst. Hautes Études Sci. Publ. Math. 32 (1967), 1361. MR 39 \#220 Zbl 0153.22301

[Grothendieck 1970] A. Grothendieck, Schémas en groupes, I: Propriétés générales des schémas en groupes, Lecture Notes in Math. 151, Springer, Berlin, 1970. MR 43 \#223a Zbl 0207.51401

[Grothendieck and Raynaud 1971] A. Grothendieck and M. Raynaud, Séminaire de Géométrie Algébrique du Bois Marie 1960/61: Revêtements étales et groupe fondamental (SGA 1), Lecture 
Notes in Mathematics 224, Springer, Berlin, 1971. Updated and annotated reprint, Soc. Math. de France, Paris, 2003. MR 50 \#7129 Zbl 0234.14002

[Hochster and Roberts 1974] M. Hochster and J. L. Roberts, "Rings of invariants of reductive groups acting on regular rings are Cohen-Macaulay", Advances in Math. 13 (1974), 115-175. MR 50 \#311 Zbl 0289.14010

[Iwanari 2009] I. Iwanari, "Logarithmic geometry, minimal free resolutions and toric algebraic stacks”, Publ. Res. Inst. Math. Sci. 45:4 (2009), 1095-1140. MR 2011f:14084 Zbl 1203.14058

[Keel and Mori 1997] S. Keel and S. Mori, "Quotients by groupoids", Ann. of Math. (2) 145:1 (1997), 193-213. MR 97m:14014 Zbl 0881.14018

[Neusel 2007] M. D. Neusel, Invariant theory, Student Mathematical Library 36, American Mathematical Society, Providence, RI, 2007. MR 2007m:13007 Zbl 1110.13001

[Satriano 2009] M. Satriano, "de Rham Theory for tame stacks and schemes with linearly reductive singularities”, 2009. arXiv 0911.2056v1

[Smith 1985] L. Smith, "On the invariant theory of finite pseudoreflection groups", Arch. Math. 44:3 (1985), 225-228. MR 87b:20018

[Tate 1997] J. Tate, "Finite flat group schemes", pp. 121-154 in Modular forms and Fermat's last theorem (Boston, MA, 1995), edited by G. Cornell et al., Springer, New York, 1997. MR 1638478 Zbl 0924.14024

[Vistoli 1989] A. Vistoli, "Intersection theory on algebraic stacks and on their moduli spaces", Invent. Math. 97:3 (1989), 613-670. MR 90k:14004 Zbl 0694.14001

Communicated by Brian Conrad

Received 2010-04-20 Revised 2010-12-26 Accepted 2011-01-24

satriano@umich.edu Department of Mathematics, University of Michigan, 530 Church St., Ann Arbor, MI 48109-1043, United States http://www-personal.umich.edu/ satriano/ 


\section{Algebra \& Number Theory}

msp.berkeley.edu/ant

\section{EDITORS}

MANAGING EDITOR

Bjorn Poonen

Massachusetts Institute of Technology

Cambridge, USA

\author{
EDITORIAL BOARD CHAIR \\ David Eisenbud \\ University of California \\ Berkeley, USA
}

\section{BOARD OF EDITORS}

Georgia Benkart

Dave Benson

Richard E. Borcherds

John H. Coates

J-L. Colliot-Thélène

Brian D. Conrad

Hélène Esnault

Hubert Flenner

Edward Frenkel

Andrew Granville

Joseph Gubeladze

Ehud Hrushovski

Craig Huneke

Mikhail Kapranov

Yujiro Kawamata

János Kollár

Yuri Manin

Barry Mazur

Philippe Michel

Susan Montgomery
University of Wisconsin, Madison, USA

University of Aberdeen, Scotland

University of California, Berkeley, USA

University of Cambridge, UK

CNRS, Université Paris-Sud, France

University of Michigan, USA

Universität Duisburg-Essen, Germany

Ruhr-Universität, Germany

University of California, Berkeley, USA

Université de Montréal, Canada

San Francisco State University, USA

Hebrew University, Israel

University of Kansas, USA

Yale University, USA

University of Tokyo, Japan

Princeton University, USA

Northwestern University, USA

Harvard University, USA

École Polytechnique Fédérale de Lausanne

University of Southern California, USA
Shigefumi Mori

Raman Parimala

Jonathan Pila

Victor Reiner

Karl Rubin

Peter Sarnak

Joseph H. Silverman

Michael Singer

Ronald Solomon

Vasudevan Srinivas

J. Toby Stafford

Bernd Sturmfels

Richard Taylor

Ravi Vakil

Michel van den Bergh

Marie-France Vignéras

Kei-Ichi Watanabe

Andrei Zelevinsky

Efim Zelmanov
RIMS, Kyoto University, Japan

Emory University, USA

University of Oxford, UK

University of Minnesota, USA

University of California, Irvine, USA

Princeton University, USA

Brown University, USA

North Carolina State University, USA

Ohio State University, USA

Tata Inst. of Fund. Research, India

University of Michigan, USA

University of California, Berkeley, USA

Harvard University, USA

Stanford University, USA

Hasselt University, Belgium

Université Paris VII, France

Nihon University, Japan

Northeastern University, USA

University of California, San Diego, USA

\section{PRODUCTION}

contact@msp.org

Silvio Levy, Scientific Editor

See inside back cover or www.jant.org for submission instructions.

The subscription price for 2012 is US \$175/year for the electronic version, and \$275/year (+\$40 shipping outside the US) for print and electronic. Subscriptions, requests for back issues from the last three years and changes of subscribers address should be sent to Mathematical Sciences Publishers, Department of Mathematics, University of California, Berkeley, CA 94720-3840, USA.

Algebra \& Number Theory (ISSN 1937-0652) at Mathematical Sciences Publishers, Department of Mathematics, University of California, Berkeley, CA 94720-3840 is published continuously online. Periodical rate postage paid at Berkeley, CA 94704, and additional mailing offices.

ANT peer review and production are managed by EditFLOW ${ }^{\circledR}$ from Mathematical Sciences Publishers.

PUBLISHED BY

mathematical sciences publishers

http://msp.org/

A NON-PROFIT CORPORATION

Typeset in IAT $_{\mathrm{E}} \mathrm{X}$

Copyright (C2012 by Mathematical Sciences Publishers 


\section{Algebra \& Number Theory}

\section{Volume $6 \quad$ No. $1 \quad 2012$}

The Chevalley-Shephard-Todd theorem for finite linearly reductive group schemes MATTHEW SATRIANO

The minimal resolution conjecture for points on del Pezzo surfaces

ROSA M. MIRÓ-ROIG and JOAN PONS-LLOPIS

$L$-series of Artin stacks over finite fields

SHENGHAO SUN

Multiplicative mimicry and improvements to the Pólya-Vinogradov inequality LEO GOLDMAKHER

Quiver Grassmannians and degenerate flag varieties

Giovanni Cerulli Irelli, Evgeny Feigin and Markus Reineke 